\title{
Variation of membrane particle-bound CD133 in cerebrospinal fluid of patients with subarachnoid and intracerebral hemorrhage
}

\author{
Tobias Bobinger, MD, ${ }^{1}$ Sebastian S. Roeder, MD, ${ }^{1}$ Maximilian I. Spruegel, MD, ${ }^{1}$ \\ Kilian Froehlich, MD, ${ }^{1}$ Vanessa D. Beuscher, MD, ${ }^{1}$ Philip Hoelter, MD, ${ }^{2}$ Hannes Lücking, MD, ${ }^{2}$ \\ Denis Corbeil, $\mathrm{PhD},{ }^{3}$ and Hagen B. Huttner, MD, $\mathrm{PhD}^{1}$
}

Departments of ${ }^{1}$ Neurology and ${ }^{2}$ Neuroradiology, Friedrich-Alexander University Erlangen (FAU); and ${ }^{3}$ Biotechnology Center (BIOTEC), Technische Universität Dresden, Germany

\begin{abstract}
OBJECTIVE Previous studies have demonstrated that human CSF contains membrane particles carrying the stem cell antigenic marker CD133 (prominin-1). Here, the authors analyzed the variation of the amount of these CD133-positive particles in the CSF of patients with subarachnoid hemorrhage (SAH) and intracerebral hemorrhage (ICH).

METHODS Consecutive CSF samples from 47 patients with SAH or ICH were compared to 14 healthy control patients. After differential ultracentrifugation of CSF, the membrane particle fraction was separated on gel electrophoresis and its CD133 content was probed by immunoblotting using the mouse monoclonal antibody 80B258 directed against human CD133. The antigen-antibody complexes were detected by chemiluminescence reagents and quantified using human Caco-2 cell extract as positive control with a standardized curve.

RESULTS As compared to healthy controls ( $6.3 \pm 0.5 \mathrm{ng}$ of bound CD133 antibody; $n=14$ ), the amount of membrane particle-associated CD133 immunoreactivities was significantly elevated in patients with SAH and ICH $(38.2 \pm 6.6 \mathrm{ng}$ and $61.3 \pm 11.0 \mathrm{ng}[\mathrm{p}<0.001]$ for SAH $[\mathrm{n}=18]$ and ICH $[\mathrm{n}=29]$, respectively). In both groups the CD133 level dropped during the first 7 days (i.e., day 5-7: SAH group, $24.6 \pm 10.1 \mathrm{ng}[\mathrm{p}=0.06]$; ICH group, $25.0 \pm 4.8 \mathrm{ng}$ [p $=0.002]$ ). Whereas changes in the amount of CD133-positive membrane particles between admission and day 5-7 were not associated with clinical outcomes in patients with ICH (modified Rankin Scale [mRS] scores $0-3,-30.9 \pm 12.8 \mathrm{ng}$ vs mRS scores $4-6,-21.8 \pm 10.7 \mathrm{ng} ; \mathrm{p}=0.239$ ), persistent elevation of CD133 in patients with SAH was related to impaired functional outcome 3 months after ictus ( $m R S$ scores $0-2,-29.9 \pm 8.1 \mathrm{ng}$ vs $\mathrm{mRS}$ scores $3-6,7.6 \pm 20.3 \mathrm{ng} ; \mathrm{p}=0.027$ ). These data are expressed as the mean \pm standard error of the mean (SEM).

CONCLUSIONS Levels of membrane particle-associated CD133 in the CSF of patients with SAH and ICH are significantly increased in comparison to healthy patients, and they decline during the hospital stay. Specifically, the persistent elevation of CD133-positive membrane particles within the first week may represent a possible surrogate measure for impaired functional outcome in patients with SAH.
\end{abstract}

https://thejns.org/doi/abs/10.3171/2019.11.JNS191861

KEYWORDS CD133; intracerebral hemorrhage; membrane particles; prominin-1; stem cells; subarachnoid hemorrhage; vascular disorders

$\mathrm{T}$ HE CSF surrounds the CNS, and its composition reflects the chemical and cellular environment. Its diagnostic potential as a "liquid biopsy" for studying CNS diseases is used in clinical practice on a regular basis..$^{18}$ Specific analysis of the CSF includes proteins such as antibodies, oligoclonal banding, or soluble proteins used for diagnosis of several neural diseases. ${ }^{16}$ Recent reports have indicated that CSF also contains small membrane particles ${ }^{7,40}$ such as exosomes or microvesicles and/ or ectosomes. ${ }^{9}$ The CSF-derived membrane particles have

ABBREVIATIONS CD133 = prominin-1; EVD = extraventricular drainage; $G C S=$ Glasgow Coma Scale; ICH = intracerebral hemorrhage; ICP = intracranial pressure; $\mathrm{mRS}=$ modified Rankin Scale; NIHSS = National Institutes of Health Stroke Scale; SAH = subarachnoid hemorrhage.

SUBMITTED July 5, 2019. ACCEPTED November 25, 2019.

INCLUDE WHEN CITING Published online January 24, 2020; DOI: 10.3171/2019.11.JNS191861. 
been shown to carry the stem cell antigenic marker CD133 (prominin-1), a cholesterol-binding pentaspan membrane glycoprotein. ${ }^{27,41}$

CD133 is expressed by various somatic stem cells, notably those found in the neural and hematopoietic system. ${ }^{3,29}$ Importantly, CD133 is not only limited to progenitors, but it is also detected in terminally differentiated cells such as glial cells (e.g., oligodendrocytes, astrocytes) and ependymal cells. ${ }^{1,11,23}$ The CD133 biology in neural tissues was recently reviewed. ${ }^{21} \mathrm{~A}$ remarkable characteristic of CD133 is its selective association with plasma membrane protrusions, irrespective of cell type..$^{12} \mathrm{CD} 133$ regulates the architecture and dynamics of cellular protrusions. ${ }^{36}$ Although the physiological function of CD133-positive membrane particles remains to be identified, recent studies have indicated the utility of CD133 in the CSF as a means to monitor the progression of gliomas and several neurodegenerative diseases. ${ }^{5,19,20}$

Whether or not membrane particle-bound CD133 is of diagnostic value in neurovascular diseases is essentially unestablished. Despite extensive research, patients suffering from severe subarachnoid hemorrhage (SAH) and intracerebral hemorrhage (ICH) are still compromised by high fatality rates and a lack of biomarkers to determine predictors of outcome. ${ }^{17,32}$ Here we analyzed for the first time consecutive CSF samples of patients with SAH and ICH, and correlated membrane particle-associated CD133 levels to clinical outcome measures.

\section{Methods}

\section{Study Design and Patient Selection}

We included patients with SAH and ICH admitted to the Neurological Intensive Care Unit of the Department of Neurology, University Hospital Erlangen, Germany, between September 2016 and March 2017. The study design was approved by the local institutional ethics review board and informed consent for sequential analysis was obtained from patients or their legal representatives prior to enrollment in the study. CSF was examined in 14 healthy patients receiving diagnostic lumbar puncture for exclusion of inflammatory disease or $\mathrm{SAH}$, and was compared to CSF obtained in 47 patients with SAH or ICH who required placement of external ventricular drains because of obstructive hydrocephalus; 18 patients with SAH and 29 patients with ICH. For the study flowchart, see Fig. 1. External ventricular drain placement into the frontal horn, ipsilateral to the $\mathrm{ICH}$, or contralateral to the $\mathrm{ICH}$ was left to the discretion of the neurosurgeon. All patients received standard medical treatment according to the current guidelines. ${ }^{17}$

CSF samples of patients with SAH and ICH were collected via extraventricular drainage (EVD) and examined every 2-3 days to exclude intraventricular infection according to hospital standards. ${ }^{6}$ After collection, CSF was examined by the hospital laboratory for basic CSF analysis (cell count, total protein via immunoturbidimetric assay) within 30 minutes. During this time 1-ml aliquots were stored for CD133 evaluation (see below). We categorized CSF samples as collected on day $0-2$, day 3-4, day 5-7, and day 8-10. Clinical data and medical information were retrieved from our institutional prospective database and included: demographic parameters; preexisting condition; history of cardiovascular diseases; clinical status on admission (National Institutes of Health Stroke Scale [NIHSS], Glasgow Coma Scale [GCS], modified Fisher Scale, and ICH score); and in-hospital parameters (mechanical ventilation, length of stay in hospital, length of EVD, lumbar drainage, and CSF infections). Diagnosis of SAH and ICH was based on CT examination (SOMATOM Definition AS +; Siemens) and evaluated by 2 experienced neuroradiologists.

The NIHSS, GCS, Hunt and Hess scale, and modified Fisher scale scores were determined by the treating and scale-trained physicians. Diagnosis of hypertension, diabetes, and coronary artery disease was established according to current criteria. ${ }^{15}$ Intracranial pressure (ICP) levels greater than $20 \mathrm{~mm} \mathrm{Hg}$ were treated with osmotic therapy (mannitol infusions and hypertonic saline); ICP was monitored throughout by EVD. CSF drainage management was accomplished according to recently published protocols with subsequent placement of a lumbar drain. ${ }^{37}$ In short: a lumbar drain was placed after clearance of intraventricular hemorrhage in the third and fourth ventricles, then the external ventricular drain was clamped and removed if no ICP crisis was observed within 24 hours. Delayed cerebral ischemia was defined by a new infarct on brain imaging that was not visible on the admission scans or by otherwise unexplained clinical deterioration. Catheter-associated CSF infections were closely monitored and treated according to published criteria. ${ }^{4}$

Functional outcome was assessed using the modified Rankin Scale (mRS) 33 months after hospital admission, either by physical examination during a follow-up visit or by a structured telephone interview with the patients or a legal representative. According to previous trials, we defined a favorable outcome in patients with SAH as an mRS score of $0-2$, and unfavorable outcome as an mRS score of 3-6. ${ }^{24}$ In patients with ICH a favorable outcome was defined as an $\mathrm{mRS}$ score of $0-3$, and unfavorable outcome as an mRS score of $4-6 .{ }^{39}$

\section{Analysis of CD133-Positive Membrane Particles}

After collection of CSF, protease inhibitor (Roche Diagnostics) was added to an aliquot $(1 \mathrm{ml})$ of CSF and stored at $-80^{\circ} \mathrm{C}$. For isolation of membrane particles, CSF samples were thawed on ice and centrifuged at $10,000 \mathrm{~g}$ for 30 minutes at $4^{\circ} \mathrm{C}$. Supernatant was collected and again centrifuged at $100,000 \mathrm{~g}$ for 60 minutes at $4^{\circ} \mathrm{C}$. The remaining pellet was resuspended in $35 \mu$ of Laemmli buffer. ${ }^{19,20}$ Samples were then subjected to immunoblotting. Human colorectal adenocarcinoma Caco-2 cells expressing CD133 were used as positive control and for establishing a standard curve. ${ }^{13,27} \mathrm{Caco}-2$ cell extracts were prepared as shown,,$^{13,20}$ and had a protein concentration of $10 \mu \mathrm{g} / \mu \mathrm{l}$ with a dilution of $1: 100$.

For immunoblotting, samples and positive controls were loaded on Tris-glycine gels (Precise 8\%; ThermoFisher) and transferred to polyvinylidene difluoride membranes (Immobilon-P membrane, $0.45 \mu \mathrm{m}$; Merck) according to standard procedures. ${ }^{10,11,19,20}$ Membranes were blocked for 1 hour in phosphate-buffered saline contain- 


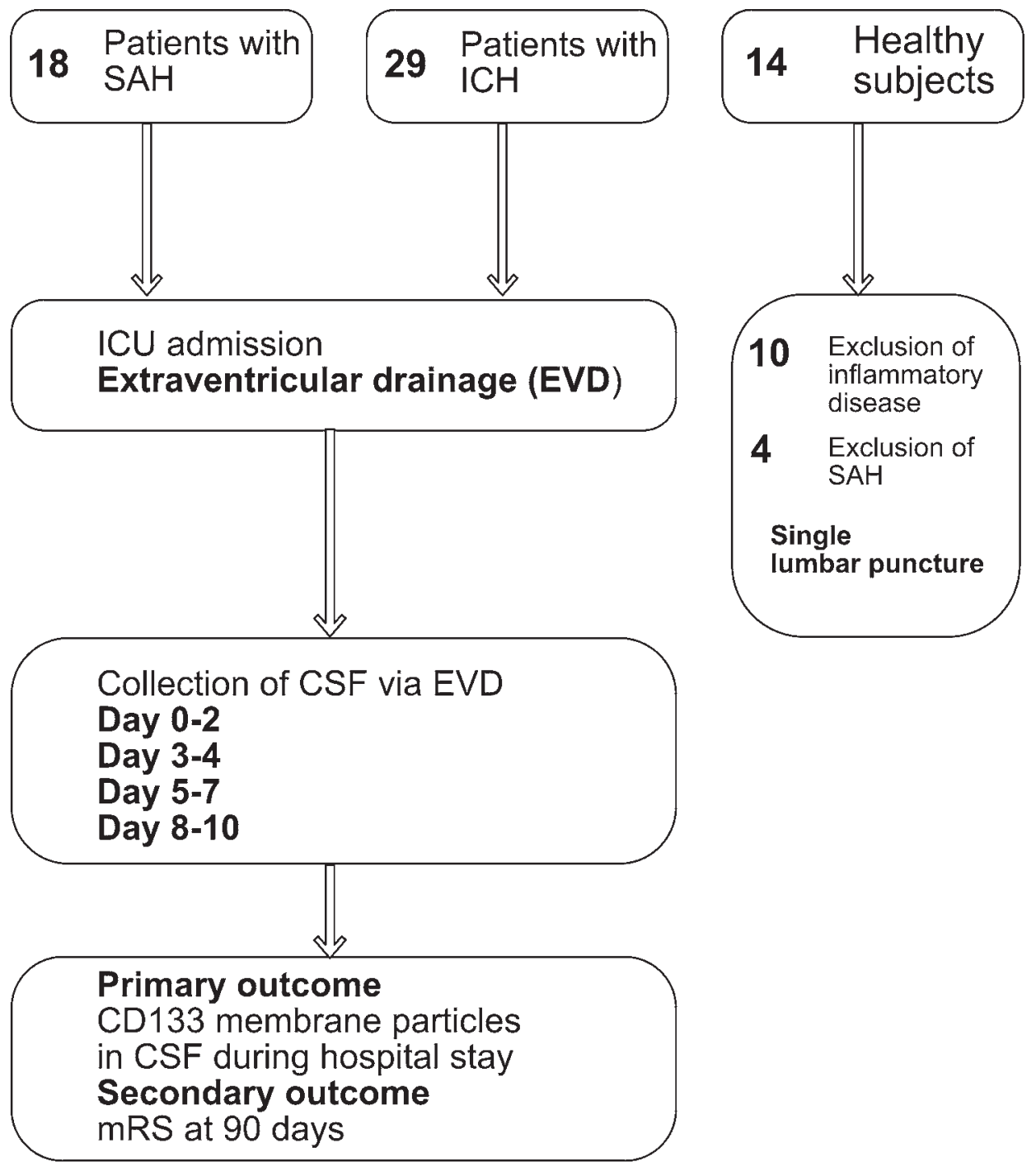

FIG. 1. Study flowchart. In this study we enrolled 14 healthy patients, 18 patients with SAH, and 29 patients with ICH. Levels of membrane particle-associated CD133 were measured on day $0-2$, day $3-4$, day $5-7$, and day $8-10$.

ing $0.3 \%$ Tween 20 and 5\% low-fat milk powder (blocking solution), and then incubated with mouse monoclonal antibody 80B258 (1 $\mu \mathrm{g} / \mathrm{ml}$; isotype IgG1) directed against the first extracellular loop of human CD133. ${ }^{20,23,28}$ The antiCD133 antibody was then diluted in blocking solution. After 12 hours, membranes were washed 3 times $(15$ minutes each) with phosphate-buffered saline containing $0.3 \%$ Tween 20 and then incubated for 60 minutes in blocking solution containing the secondary antibody: horseradish peroxidase-conjugated goat anti-mouse (1:5000; Jackson Immunoresearch Laboratories). Enhanced chemiluminescence reagents (SuperSignal West Femto Substrate; ThermoFisher) was used to detect the antigen-antibody complexes. Visualization of the complexes was performed with the image reader Fusion FX 7 Spectra (Vilber Lourmat $\mathrm{GmbH}$ ), as reported recently.5,19 Quantification was calculated using Image Quant TL (GE Healthcare GmbH). Caco-2 extracts were used as an internal standard sample and evaluated on each blot. The amount of bound monoclonal antibody 80B258 (in nanograms) was calculated using an established standard curve of Caco-2 cell extracts, as described. $5,19,20$

\section{Statistical Analysis}

For statistical analysis the IBM SPSS Statistics software version 22.0 (IBM Corp.) and GraphPad Prism 8.0 (GraphPad Inc.) were used. The Kolmogorov-Smirnov test was performed to determine the data distribution. Normally distributed data are presented as the mean \pm standard error of the mean (SEM) and compared using the Student t-test. Other data are presented as the median and range, and compared using the Mann-Whitney U-test (unpaired data) or the Wilcoxon signed-rank test (paired data). The Pearson chi-square and the Fisher exact tests were used to compare frequency of categorized variables, and the Kruskal-Wallis test was used for comparison of 
TABLE 1. Baseline characteristics and in-hospital measures and complications in 18 patients with SAH stratified by outcome after 3 months

\begin{tabular}{|c|c|c|c|}
\hline \multirow[b]{2}{*}{ Characteristic } & \multicolumn{2}{|c|}{ Outcome Group } & \multirow[b]{2}{*}{$\mathrm{p}$ Value } \\
\hline & $m R S 0-2, n=10$ & $m R S 3-6, n=8$ & \\
\hline Age, yrs & $55.0(48.8-60.3)$ & $58.0(44.8-62.8)$ & 0.696 \\
\hline Female sex & $6(60 \%)$ & $6(75 \%)$ & 0.638 \\
\hline \multicolumn{4}{|l|}{ Previous comorbidities } \\
\hline Hypertension & $5(50 \%)$ & $3(37.5 \%)$ & 0.664 \\
\hline Diabetes mellitus & $1(10 \%)$ & $1(12.5 \%)$ & $>0.99$ \\
\hline History of smoking & $5(50 \%)$ & $4(50 \%)$ & $>0.99$ \\
\hline \multicolumn{4}{|l|}{ Admission status } \\
\hline GCS score & $12(3-15)$ & $3(3-3)$ & 0.033 \\
\hline Hunt \& Hess scale score & $3(1-4)$ & $5(3-5)$ & 0.034 \\
\hline Modified Fisher scale score & $4(3-4)$ & $4(4-4)$ & 0.573 \\
\hline \multicolumn{4}{|c|}{ In-hospital measures \& complications } \\
\hline Duration of ventilation, days & $9.0(1.8-20.8)$ & $14.0(9.0-31.0)$ & 0.121 \\
\hline Duration of EVD, days & $6.0(5.8-8.3)$ & $10.0(6.0-15.0)$ & 0.088 \\
\hline Lumbar drainage & $10(100 \%)$ & $6(75 \%)$ & 0.183 \\
\hline Osmotherapy & $2(20 \%)$ & $6(75 \%)$ & 0.054 \\
\hline Ventriculitis & $0(0.0 \%)$ & $1(12.5 \%)$ & 0.444 \\
\hline Delayed cerebral ischemia & $2(20 \%)$ & $5(62.5 \%)$ & 0.145 \\
\hline Length of stay, days & $23.5(20.0-30.3)$ & $24.5(9.8-36.5)$ & 0.965 \\
\hline
\end{tabular}

Boldface type indicates statistical significance at $p<0.05$. Values are expressed as the number of patients (\%) or as the median (IQR; 25 th- 75 th percentile). An mRS score of $0-2$ denotes a favorable outcome; an mRS score of 3-6 denotes an unfavorable outcome.

several independent groups. The relationship between particle-associated CD133 and cell count and protein levels in CSF was investigated by linear regression. All statistical tests were 2-sided, the significance level was set at $\alpha=$ 0.05 , and findings were corrected for multiple comparisons by Holm's sequential Bonferroni procedure.

\section{Results \\ CD133-Positive Membrane Particles Are Increased in Patients With $\mathrm{SAH}$ and $\mathrm{ICH}$}

To investigate the potential variation of membrane particle-associated CD133 levels in CSF samples of patients with SAH and $\mathrm{ICH}$, we examined 47 patients with intracranial hemorrhages and compared them with 14 healthy individuals who received a diagnostic lumbar puncture for exclusion of inflammatory disease or SAH (Fig. 1). The characteristics of all enrolled patients with SAH or ICH are summarized in Tables 1 and 2, stratified by favorable and unfavorable outcome after 3 months. As compared to healthy controls (median age 53.0 years [IQR 48.3-60.0 years]; $50 \%$ female), the amount of membrane particle-associated CD133 - as observed by CD133 immunoreactivity (see Methods) on first CSF analysis (i.e., day 0-2) - was significantly increased in patients with SAH and ICH $(6.3$ $\pm 0.5 \mathrm{ng}$ bound anti-CD133 antibody in healthy controls vs $38.2 \pm 6.6 \mathrm{ng}$ and $61.3 \pm 11.0 \mathrm{ng}$ for SAH [n $=18]$ and ICH [ $\mathrm{n}=29$ ], respectively; Kruskal-Wallis test, $\mathrm{p}<0.001$; Figs. 2 and 3, upper panels). Interestingly, linear regression analysis revealed no correlation between the amount of CD133 immunoreactivity and total protein levels on day $0-2\left(r^{2}=0.024 ; p=0.259\right)$ or CSF cell count on day $0-2\left(r^{2}=0.077 ; p=0.152\right)$, suggesting that membrane particle-associated CD133 might represent a novel biomarker of these diseases.

The amount of CD133-positive membrane particles decreased in subsequent CSF analyses during the course of patients' stay. In both groups the amount of CD133 dropped significantly when comparing results on day $0-2$ after admission (see preceding paragraph) to day 3-4 (Fig. 2, upper panel-SAH: day 3-4, $20.0 \pm 3.3 \mathrm{ng}, \mathrm{p}=$ 0.033 ; Fig. 3, upper panel-ICH: day 3-4, $26.2 \pm 4.2 \mathrm{ng}, \mathrm{p}$ $<0.001$ ) and on day 8-10 (SAH: $10.4 \pm 4.7 \mathrm{ng}, \mathrm{p}=0.004$; and ICH: $20.0 \pm 4.4 \mathrm{ng}, \mathrm{p}<0.001)$.

\section{Variation of the Amount of CD-133-Positive Membrane Particles and Association With Clinical Outcomes}

The amount of membrane particle-associated CD133 on admission did not distinguish between patients with favorable and unfavorable outcome in both entities (patients with SAH: favorable outcome, $39.1 \pm 7.8 \mathrm{ng}$ vs unfavorable outcome, $36.4 \pm 13.3 \mathrm{ng}, \mathrm{p}=0.797$; patients with ICH: favorable outcome, $50.8 \pm 18.7 \mathrm{ng}$ vs unfavorable outcome, $65.2 \pm 14.4, p=0.549$; Figs. 2 and 3, lower panels, respectively).

Comparing patients with $\mathrm{SAH}$ who had a favorable versus unfavorable functional outcome revealed that CD133 immunoreactivity levels showed a persistent elevation in those patients with an unfavorable outcome (i.e., difference of CD133 between day 0-2 vs day 5-7: favorable outcome, $-29.9 \pm 8.1 \mathrm{ng}$ vs unfavorable outcome, $7.6 \pm$ $20.3 \mathrm{ng} ; \mathrm{p}=0.027$ ). In our cohort, patients with persistent elevation of CD133-positive membrane particles showed a 
TABLE 2. Baseline characteristics, admission status, imaging characteristics, in-hospital measures, and complications in 28 patients with ICH stratified by outcome after 3 months*

\begin{tabular}{|c|c|c|c|}
\hline \multirow[b]{2}{*}{ Characteristic } & \multicolumn{2}{|c|}{ Outcome Group } & \multirow[b]{2}{*}{$\mathrm{p}$ Value } \\
\hline & $m R S 0-3, n=5$ & $m R S 4-6, n=23$ & \\
\hline Age, yrs & $57.0(43.0-66.5)$ & $74.0(61.0-80.0)$ & 0.033 \\
\hline Female sex & $1(20 \%)$ & $7(30.4 \%)$ & $>0.99$ \\
\hline \multicolumn{4}{|l|}{ Previous comorbidities } \\
\hline Premorbid mRS score & $0(0-1)$ & $2(0-3)$ & 0.071 \\
\hline Hypertension & $4(80 \%)$ & $22(95.7 \%)$ & 0.331 \\
\hline Diabetes mellitus & $1(20 \%)$ & $11(47.8 \%)$ & 0.355 \\
\hline Dyslipidemia & $2(40 \%)$ & $6(26.1 \%)$ & 0.606 \\
\hline Coronary artery disease & $1(20 \%)$ & $7(30.4 \%)$ & $>0.99$ \\
\hline Atrial fibrillation & $0(0.0 \%)$ & $10(43.5 \%)$ & 0.128 \\
\hline Previous ischemic stroke & $2(40 \%)$ & $5(21.7 \%)$ & 0.574 \\
\hline Previous hemorrhagic stroke & $0(0.0 \%)$ & $1(4.4 \%)$ & $>0.99$ \\
\hline Oral anticoagulation & $0(0.0 \%)$ & $4(17.4 \%)$ & 0.568 \\
\hline \multicolumn{4}{|l|}{ Admission status } \\
\hline GCS score & $13(6-15)$ & $5(3-10)$ & 0.037 \\
\hline NIHSS score & $7(3-11)$ & $15(13-38)$ & 0.041 \\
\hline $\mathrm{ICH}$ score & $1(1-1)$ & $2(2-3)$ & 0.089 \\
\hline \multicolumn{4}{|l|}{ Imaging characteristics } \\
\hline Initial ICH vol, ml & $23.5(20.5-32.0)$ & $44.0(37.0-50.0)$ & 0.002 \\
\hline Intraventricular hemorrhage & $4(80.0 \%)$ & $19(82.6 \%)$ & $>0.99$ \\
\hline \multicolumn{4}{|c|}{ In-hospital measures \& complications } \\
\hline Duration of ventilation, days & $3.0(2.0-17.5)$ & $16.0(10.0-22.0)$ & 0.107 \\
\hline Duration of EVD, days & $10.0(6.0-22.5)$ & $11.0(7.8-13.0)$ & 0.786 \\
\hline Osmotherapy & $3(60.0 \%)$ & $14(60.9 \%)$ & $>0.99$ \\
\hline Ventriculitis & $0(0.0 \%)$ & $1(4.4 \%)$ & $>0.99$ \\
\hline Intraventricular fibrinolysis & $2(40.0 \%)$ & $12(52.2 \%)$ & $>0.99$ \\
\hline Length of stay, days & $23.0(14.5-32.0)$ & $16.0(14.0-25.0)$ & 0.483 \\
\hline
\end{tabular}

Boldface type indicates statistical significance at $p<0.05$. Values are expressed as the number of patients (\%) or as the median (IQR; 25th-75th percentile). An mRS score of 0 -3 denotes a favorable outcome; an mRS score of 4-6 denotes an unfavorable outcome.

* One patient in the $\mathrm{ICH}$ group was lost to follow-up and therefore was excluded from outcome-related analysis.

high rate of delayed cerebral ischemia; although without significant difference $(62.5 \%$ [5/8] vs $20.0 \%$ [2/10]; $\mathrm{p}=$ $0.145)$.

Comparing patients with ICH who had favorable versus unfavorable functional outcome revealed that CD133 immunoreactivity levels dropped to a similar extent in patients with both favorable and unfavorable outcome (i.e., reduction of CD133 between day $0-2$ vs day 5-7: favorable outcome, $-30.9 \pm 12.8 \mathrm{ng}$ vs unfavorable outcome, $-21.8 \pm 10.7 \mathrm{ng})$.

\section{Discussion}

The present study for the first time systematically investigated human CSF for membrane particle-associated CD133 in patients with SAH and ICH. In essence, compared to healthy individual controls, patients with SAH and ICH showed markedly increased levels of CD133, which declined during the hospital stay. Although the amount of CD133 and its dynamic did not differentiate favorable and unfavorable outcome in ICH, persistent el- evation was linked to unfavorable outcome in SAH. Some aspects deserve further attention.

Analysis of CSF is often used as a diagnostic tool in patients with SAH or ICH. Several proteins in CSF have been examined to constitute a possible prognostic factor for outcome in these diseases. ${ }^{35,38}$ Analysis of membraneassociated particles represents an interesting tool for monitoring neurological disease because cell membrane dynamics are tracked, which may shed light on the severity of the disease. ${ }^{2}$ The key question remains: why is a persistent or second elevation of CD133 levels linked to functional outcome?

The easiest explanation would be a surrogate of disease severity being less pronounced in those patients with fast CD133 decrease and thus more favorable outcome. A possible source of CD133-positive membrane particles are ependymal cells and/or astrocytes residing in the subventricular zone. ${ }^{1}$ These cells protrude with their CD133-positive cilium into the lumen, and may be the source of these membrane particles. ${ }^{11,25,27}$ Even though current research did not prove any evidence of infiltrating cells contribut- 

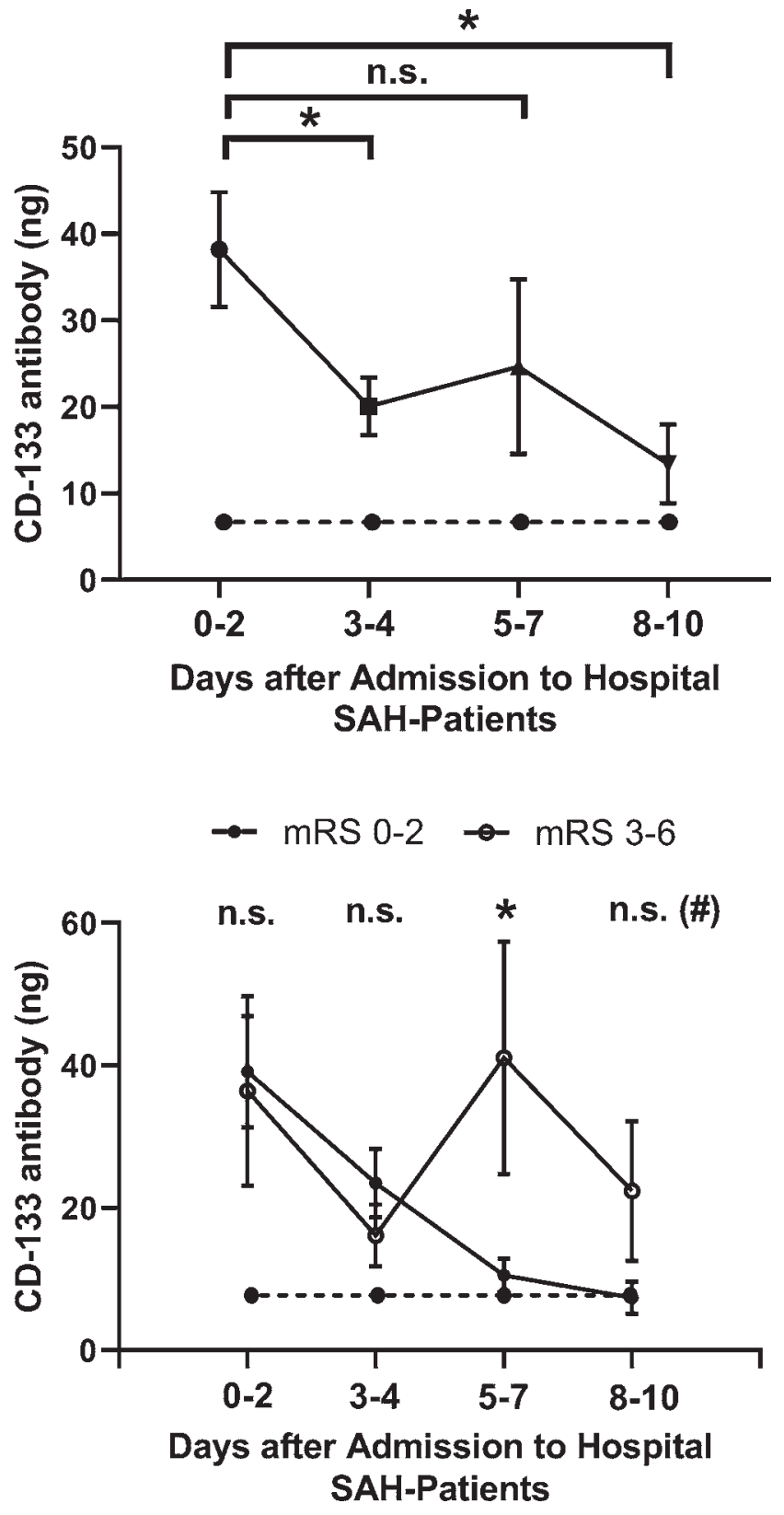

FIG. 2. Graphs showing amount of CD133-positive membrane particles in patients with SAH. The dashed line illustrates the amount of CD133 particles in a healthy cohort. Upper: The graph depicts the overall course of CD133-positive membrane particles for all patients (solid line) as revealed by CD133 immunoreactivity (for quantification see Methods section) at day $0-2(n=18$, circle); day $3-4(n=17$, square); day $5-7$ ( $n$ $=16$, up-pointing triangle); and day 8-10 ( $\mathrm{n}=13$, down-pointing triangle) by comparison to healthy patients. The amount of CD133 was significantly lower on day $3-4(p=0.033)$ and day $8-10(p=0.004)$ compared to the first measurement. No significant difference (n.s.) was found on day 5-7 ( $p=0.064)$. Lower: Graph showing findings stratified by favorable (mRS scores $0-2$ ) and unfavorable (mRS scores 3-6) outcome 3 months after admission to the hospital. Analysis revealed a significantly elevated amount of CD133 particles in patients with unfavorable outcome on day 5-7. Day 0-2: $p=0.797$; day 3-4: $p=0.397$; day $5-7: p=$ 0.032; day 8-10: $p=0.257$. \# = not significant after Holm's Bonferroni correction.

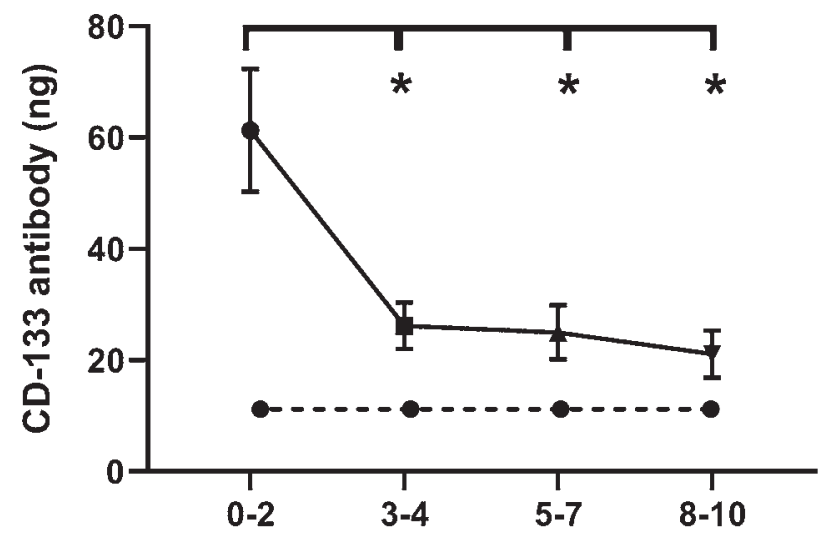

\section{Days after Admission to Hospital ICH-Patients}

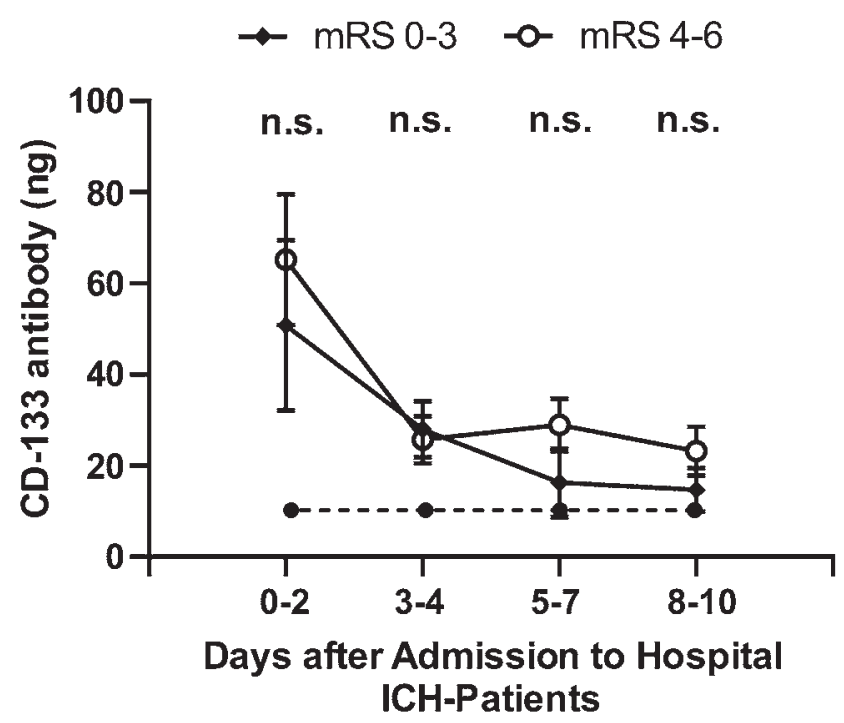

FIG. 3. Amount of CD133-positive membrane particles in patients with $\mathrm{ICH}$. The dashed line illustrates the amount of CD133 particles in a healthy cohort. Upper: The graph presents the amount of CD133-positive membrane particles for all patients (solid line) as revealed by CD133 immunoreactivity (for quantification see Methods section) at day 0-2 $(n=29$, circle); day 3-4 $(n=26$, square); day 5-7 $(n=24$, up-pointing triangle); and day 8-10 ( $\mathrm{n}=21$, down-pointing triangle) by comparison to healthy patients. The amount of CD133 was significantly lower on day 3-4 ( $p<0.001)$, day 5-7 $(p=0.002)$, and day 8-10 ( $p<0.001)$ compared to the first measurement. Lower: Graph showing findings stratified by favorable (mRS scores 0-3) and unfavorable (mRS scores 4-6) outcome 3 months after admission to the hospital. No significant difference is observed between the groups. Day $0-2: p=0.549$; day $3-4: p=$ 0.442 ; day $5-7: p=0.365$; day $8-10: p=0.661$. 
ing to CD133-positive membrane particles in the CSF, it cannot be ruled out. Cellular damage in patients with SAH and ICH may explain the excess of membrane particles in the CSF.

CD133 is found in myelin, ${ }^{11}$ and initial damage or secondary remodeling processes of myelin sheaths might contribute to the levels of CD133 in CSF. ${ }^{11,30} \mathrm{~A}$ recent report has suggested that $\mathrm{CD133}$ plays a role in myelination. ${ }^{8}$ Moreover, an increased amount of CD133-positive membrane particles have been found in patients with neurodegenerative diseases as well as multiple sclerosis. ${ }^{5} \mathrm{Cy}-$ tokines trigger a cascade of inflammatory processes, leading to destruction of myelin, axons, and oligodendrocytes. Subsequently, trophic factors initiate a modulation of the blood-brain barrier and the extracellular matrix..14,26,31 Both processes may release CD133-positive membrane particles into the CSF and might explain increased levels. The connection between inflammation and increased CD133 has been proposed in solid cancer. ${ }^{22,34}$ Future studies need to evaluate both processes-inflammation and subsequent gliogenesis - and its contribution to the elevated CD133 levels in the CSF.

Our study has a number of shortcomings: 1) our results might be biased due to the semiquantitative method of immunoblotting and the lack of more robust methods such as enzyme-linked immunosorbent assay (ELISA); 2) the number of patients is low-further studies need to verify our results in a larger cohort of patients; and 3) the full characterization of our membrane particles (e.g., ectosomes vs exosomes) is lacking. The immune isolation of CSF-associated CD133-positive membrane particles as recently performed for particles found in saliva, and the exhaustive analysis of their content (proteins, lipids, and/or nucleic acids) may reveal their precise origin, and highlights alternative and/or additional biomarkers. Nonetheless, our study is the first to evaluate CD133-positive membrane particles in patients with SAH and ICH, and emphasizes the need for further analyses.

\section{Conclusions}

Compared to healthy individual controls, levels of membrane particle-associated CD133 in the CSF of patients with ICH are significantly increased, and decline during the hospital stay. Notably, patients with SAH who have a persistence of higher amounts of CD133 demonstrate impaired outcome, indicating a possible surrogate of functional status.

\section{Acknowledgments}

We thank Ulrike Naumann for her excellent technical assistance.

\section{References}

1. Bachor TP, Karbanová J, Büttner E, Bermúdez V, MarquioniRamella M, Carmeliet P, et al: Early ciliary and prominin-1 dysfunctions precede neurogenesis impairment in a mouse model of type 2 diabetes. Neurobiol Dis 108:13-28, 2017

2. Bannai H: Molecular membrane dynamics: insights into synaptic function and neuropathological disease. Neurosci Res 129:47-56, 2018
3. Bauer N, Wilsch-Bräuninger M, Karbanová J, Fonseca AV, Strauss D, Freund D, et al: Haematopoietic stem cell differentiation promotes the release of prominin-1/CD133-containing membrane vesicles - a role of the endocytic-exocytic pathway. EMBO Mol Med 3:398-409, 2011

4. Beer R, Pfausler B, Schmutzhard E: Management of nosocomial external ventricular drain-related ventriculomeningitis. Neurocrit Care 10:363-367, 2009

5. Bobinger T, May L, Lücking H, Kloska SP, Burkardt P, Spitzer P, et al: CD133-positive membrane particles in cerebrospinal fluid of patients with inflammatory and degenerative neurological diseases. Front Cell Neurosci 11:77, 2017

6. Champey J, Mourey C, Francony G, Pavese P, Gay E, Gergele $\mathrm{L}$, et al: Strategies to reduce external ventricular drain-related infections: a multicenter retrospective study. J Neurosurg 130:2034-2039, 2019

7. Chiasserini D, van Weering JR, Piersma SR, Pham TV, Malekzadeh A, Teunissen CE, et al: Proteomic analysis of cerebrospinal fluid extracellular vesicles: a comprehensive dataset. J Proteomics 106:191-204, 2014

8. Choi MH, Na JE, Yoon YR, Rhyu IJ, Ko YG, Baik JH: Hypomyelination and cognitive impairment in mice lacking CD133 (prominin-1). Biochem Biophys Res Commun 502:291-298, 2018

9. Colombo M, Raposo G, Théry C: Biogenesis, secretion, and intercellular interactions of exosomes and other extracellular vesicles. Annu Rev Cell Dev Biol 30:255-289, 2014

10. Corbeil D, Fargeas CA, Huttner WB: Rat prominin, like its mouse and human orthologues, is a pentaspan membrane glycoprotein. Biochem Biophys Res Commun 285:939-944, 2001

11. Corbeil D, Joester A, Fargeas CA, Jászai J, Garwood J, Hellwig A, et al: Expression of distinct splice variants of the stem cell marker prominin-1 (CD133) in glial cells. Glia 57:860874,2009

12. Corbeil D, Röper K, Fargeas CA, Joester A, Huttner WB: Prominin: a story of cholesterol, plasma membrane protrusions and human pathology. Traffic 2:82-91, 2001

13. Corbeil D, Röper K, Hellwig A, Tavian M, Miraglia S, Watt SM, et al: The human AC133 hematopoietic stem cell antigen is also expressed in epithelial cells and targeted to plasma membrane protrusions. J Biol Chem 275:5512-5520, 2000

14. Cristofanilli M, Cymring B, Lu A, Rosenthal H, Sadiq SA: Cerebrospinal fluid derived from progressive multiple sclerosis patients promotes neuronal and oligodendroglial differentiation of human neural precursor cells in vitro. Neuroscience 250:614-621, 2013

15. Cryer MJ, Horani T, DiPette DJ: Diabetes and hypertension: a comparative review of current guidelines. J Clin Hypertens (Greenwich) 18:95-100, 2016

16. Gastaldi M, Zardini E, Leante R, Ruggieri M, Costa G, Cocco E, et al: Cerebrospinal fluid analysis and the determination of oligoclonal bands. Neurol Sci 38 (Suppl 2):217-224, 2017

17. Hemphill JC III, Greenberg SM, Anderson CS, Becker K, Bendok BR, Cushman M, et al: Guidelines for the management of spontaneous intracerebral hemorrhage: a guideline for healthcare professionals from the American Heart Association/American Stroke Association. Stroke 46:2032-2060, 2015

18. Hühmer AF, Biringer RG, Amato H, Fonteh AN, Harrington MG: Protein analysis in human cerebrospinal fluid: physiological aspects, current progress and future challenges. Dis Markers 22:3-26, 2006

19. Huttner HB, Corbeil D, Thirmeyer C, Coras R, Köhrmann $\mathrm{M}$, Mauer C, et al: Increased membrane shedding_indicated by an elevation of CD133-enriched membrane particles-into the CSF in partial epilepsy. Epilepsy Res 99:101-106, 2012

20. Huttner HB, Janich P, Köhrmann M, Jászai J, Siebzehnrubl F, Blümcke I, et al: The stem cell marker prominin-1/CD133 on 
membrane particles in human cerebrospinal fluid offers novel approaches for studying central nervous system disease.

Stem Cells 26:698-705, 2008

21. Jászai J, Corbeil D, Fargeas CA: Comprehensive overview of CD133 biology in neural tissues across species, in Pruszak J (ed): Neural Surface Antigens: From Basic Biology Towards Biomedical Applications. London: Elsevier, 2015, pp $113-129$

22. Karbanová J, Laco J, Marzesco AM, Janich P, Voborníková M, Mokrý J, et al: Human prominin-1 (CD133) is detected in both neoplastic and non-neoplastic salivary gland diseases and released into saliva in a ubiquitinated form. PLoS One 9:e98927, 2014

23. Karbanová J, Missol-Kolka E, Fonseca AV, Lorra C, Janich P, Hollerová $\mathrm{H}$, et al: The stem cell marker CD133 (prominin-1) is expressed in various human glandular epithelia. J Histochem Cytochem 56:977-993, 2008

24. Kirkpatrick PJ, Turner CL, Smith C, Hutchinson PJ, Murray GD: Simvastatin in aneurysmal subarachnoid haemorrhage (STASH): a multicentre randomised phase 3 trial. Lancet Neurol 13:666-675, 2014

25. Kriegstein A, Alvarez-Buylla A: The glial nature of embryonic and adult neural stem cells. Annu Rev Neurosci 32:149-184, 2009

26. Ludwin SK, Rao VTs, Moore CS, Antel JP: Astrocytes in multiple sclerosis. Mult Scler 22:1114-1124, 2016

27. Marzesco AM, Janich P, Wilsch-Bräuninger M, Dubreuil V, Langenfeld K, Corbeil D, et al: Release of extracellular membrane particles carrying the stem cell marker prominin-1 (CD133) from neural progenitors and other epithelial cells. J Cell Sci 118:2849-2858, 2005

28. Missol-Kolka E, Karbanová J, Janich P, Haase M, Fargeas CA, Huttner WB, et al: Prominin-1 (CD133) is not restricted to stem cells located in the basal compartment of murine and human prostate. Prostate 71:254-267, 2011

29. Mizrak D, Brittan M, Alison M: CD133: molecule of the moment. J Pathol 214:3-9, 2008

30. Muramatsu R, Takahashi C, Miyake S, Fujimura H, Mochizuki H, Yamashita T: Angiogenesis induced by CNS inflammation promotes neuronal remodeling through vesselderived prostacyclin. Nat Med 18:1658-1664, 2012

31. Nait-Oumesmar B, Picard-Riéra N, Kerninon C, Baron-Van Evercooren A: The role of SVZ-derived neural precursors in demyelinating diseases: from animal models to multiple sclerosis. J Neurol Sci 265:26-31, 2008

32. Pegoli M, Mandrekar J, Rabinstein AA, Lanzino G: Predictors of excellent functional outcome in aneurysmal subarachnoid hemorrhage. J Neurosurg 122:414-418, 2015

33. Rankin J: Cerebral vascular accidents in patients over the age of 60. II. Prognosis. Scott Med J 2:200-215, 1957

34. Rappa G, Fargeas CA, Le TT, Corbeil D, Lorico A: Letter to the editor: An intriguing relationship between lipid droplets, cholesterol-binding protein CD133 and Wnt/ $\beta$-catenin signaling pathway in carcinogenesis. Stem Cells 33:1366-1370, 2015

35. Roy-O'Reilly M, Zhu L, Atadja L, Torres G, Aronowski J, McCullough L, et al: Soluble CD163 in intracerebral hemorrhage: biomarker for perihematomal edema. Ann Clin Transl Neurol 4:793-800, 2017
36. Singer D, Thamm K, Zhuang H, Karbanová J, Gao Y, Walker JV, et al: Prominin-1 controls stem cell activation by orchestrating ciliary dynamics. EMBO J 38:e99845, 2019

37. Staykov D, Kuramatsu JB, Bardutzky J, Volbers B, Gerner ST, Kloska SP, et al: Efficacy and safety of combined intraventricular fibrinolysis with lumbar drainage for prevention of permanent shunt dependency after intracerebral hemorrhage with severe ventricular involvement: a randomized trial and individual patient data meta-analysis. Ann Neurol 81:93-103, 2017

38. Van Dreden P, Hue G, Dreyfus JF, Woodhams B, Vasse M: Procoagulant phospholipids and tissue factor activity in cerebrospinal fluid from patients with intracerebral haemorrhage. Adv Hematol 2014:576750, 2014

39. Wartenberg KE, Wang X, Muñoz-Venturelli P, Rabinstein AA, Lavados PM, Anderson CS, et al: intensive care unit admission for patients in the INTERACT2 ICH blood pressure treatment trial: characteristics, predictors, and outcomes. Neurocrit Care 26:371-378, 2017

40. Welton JL, Loveless S, Stone T, von Ruhland C, Robertson NP, Clayton A: Cerebrospinal fluid extracellular vesicle enrichment for protein biomarker discovery in neurological disease; multiple sclerosis. J Extracell Vesicles 6:1369805, 2017

41. Wiklander OPB, Bostancioglu RB, Welsh JA, Zickler AM, Murke F, Corso G, et al: Systematic methodological evaluation of a multiplex bead-based flow cytometry assay for detection of extracellular vesicle surface signatures. Front Immunol 9:1326, 2018

\section{Disclosures}

The study was not funded. The authors report no conflict of interest concerning the materials or methods used in this study or the findings specified in this paper.

\section{Author Contributions}

Conception and design: Bobinger, Corbeil, Huttner. Acquisition of data: Bobinger, Roeder, Huttner. Analysis and interpretation of data: Bobinger, Roeder, Sprügel, Froehlich, Lücking, Corbeil, Huttner. Drafting the article: Bobinger, Froehlich, Beuscher, Corbeil, Huttner. Critically revising the article: Roeder, Sprügel, Froehlich, Beuscher, Hoelter, Corbeil, Huttner. Reviewed submitted version of manuscript: all authors. Approved the final version of the manuscript on behalf of all authors: Bobinger. Statistical analysis: Bobinger, Roeder. Administrative/technical/material support: Huttner. Study supervision: Huttner.

\section{Correspondence}

Tobias Bobinger: Friedrich-Alexander University Erlangen (FAU), Erlangen, Germany. tobias.bobinger@uk-erlangen.de. 